\title{
Shape-preserving and reactive adaptation of robot end-effector trajectories
}

\author{
Maxim Vochten, Wilm Decré, Erwin Aertbeliën, and Joris De Schutter
}

\begin{abstract}
Variation in task requirements and operation in uncertain environments require robots that can continuously adapt their motions during execution. To do this successfully, the original motion characteristics must be preserved. This paper presents an approach for on-line adaptation of endeffector trajectories for robot manipulators. The trajectory generator aims to preserve the shape of a given model trajectory through its coordinate-invariant shape description. In addition, this trajectory generator is coupled to a task specification and control framework that enables reactive behavior of the robot. Simulation results on the UR10 robot indicate the capability of the developed approach to generalize end-effector trajectories towards new targets while preserving the original trajectory shape. The controller's reactivity is shown towards moving targets and obstacles in the environment. The combination of shape preservation and reactive control may endow robots with better on-line motion generalization capabilities.
\end{abstract}

\section{INTRODUCTION}

Future robots are expected to autonomously perform a multitude of varied tasks in industrial, domestic and health care environments. To successfully deal with varying task requirements in varying environments, online adaptations of the robot's end-effector motions are required that preserve as much as possible the characteristics of the original motion. In particular, this paper focuses on end-effector trajectories involving general rigid-body motions, such as pouring a jug, scooping with a spoon or handing over objects.

Trajectory representation is one of the main factors that can limit the adaptation of robot trajectories. To program new human-like motions on the robot, the Learning by Demonstration (LbD) approach [1], [2] is often used to rapidly build a motion model starting from one or several human demonstrations. These motion models typically depend on the specific context in which the demonstrations were recorded, such as the choice for the coordinate reference frames. These choices limit the ability to generalize, resulting in more and more of the motion characteristics that are lost when moving farther away from the original demonstrations. Therefore, there is a need for suitably generalizable motion models that can be constructed from a few demonstrations.

\section{A. Objectives and approach}

The objective of this paper is to start from a given model for the nominal robot end-effector trajectory and generate

This work was supported by ERC Advanced Grant: ROBOTGENSKILL and Flanders Make Project PROUD_SBO.

All authors are with the Department of Mechanical Engineering, KU Leuven \& Flanders Make Core Lab ROB, 3001 Leuven, Belgium. (email: maxim.vochten@kuleuven.be, wilm.decre@kuleuven.be, erwin.aertbelien@kuleuven.be, joris.deschutter@kuleuven.be) new trajectories that retain as much as possible the shape of the original trajectory, even when far away in Cartesian space. During execution, certain task constraints such as the target pose may change and therefore the generated trajectories require on-line adaptability. The robot controller should track the trajectory, but at the same time be able to deal with platform and environmental constraints, such as joint limit avoidance, collision avoidance and force interaction.

To enable shape-preserving trajectory adaptation, we follow the approach of our prior work [3] in which demonstrated motion trajectories are first represented using coordinate-invariant shape descriptors. This approach produces motion models that are independent of the context in which the motion was demonstrated, allowing for a better generalization. The main limitation of the method is that calculating new trajectories is computationally expensive, prohibiting its use in on-line applications. To resolve this limitation, this paper introduces a two-step approach. In the first step, the optimal adaptation of trajectories based on their shape descriptor is reformulated using a locally receding window, allowing for on-line adaptation. This trajectory generator is coupled in a second step with a reactive controller based on our task specification and control framework $e T a S L$ [4]. This framework enables the specification of reactive behaviors in a modular way. From these specifications, an appropriate controller with good tracking behavior can be automatically derived. The main challenge for the integration of the trajectory generator into eTaSL is the determination of the progress along the trajectory to select the appropriate position and velocity setpoints for the controller and at the same time select the correct initial values for the trajectory generator.

The developed approach is depicted in Figure 1 and consists of a cascaded control loop. The outer loop adapts the trajectory in a shape-preserving way corresponding to new trajectory constraints and operates at a lower frequency. The inner loop operates at a higher frequency and features a reactive controller in eTaSL that follows the generated trajectory in combination with other specified reactive behaviors. The reactive controller feeds back the current progress of the robot to the trajectory generator so that new trajectories are calculated from the current location.

\section{B. Contributions and outline}

The contributions of this paper are twofold. The first contribution is a new local receding window algorithm for online adaptation of rigid-body motion trajectories, as opposed to the global optimization approach in our prior work [3]. 


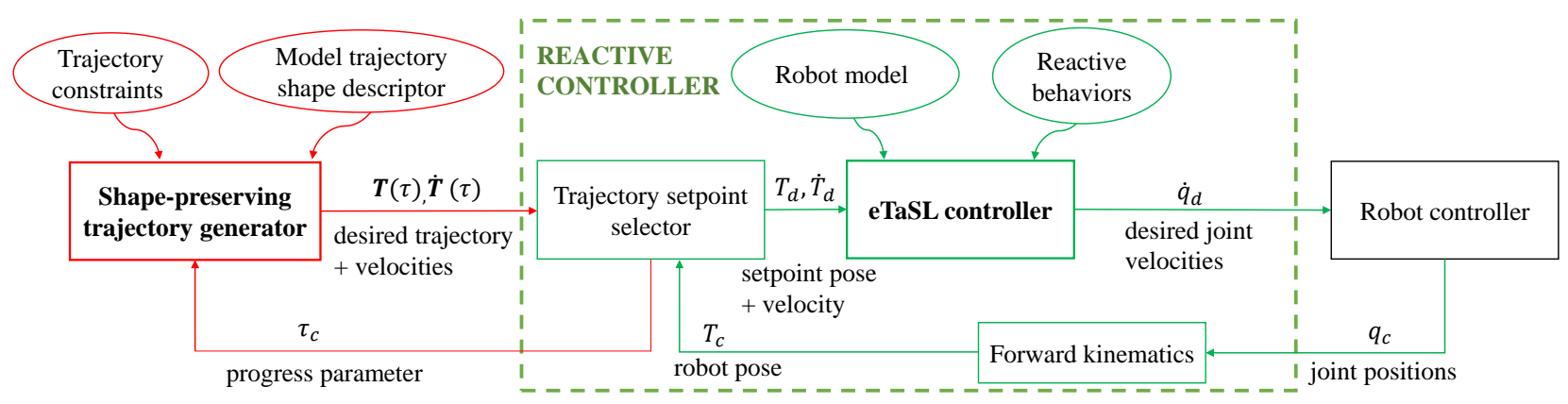

Fig. 1: Proposed approach for shape-preserving trajectory adaptation and control. The trajectory generator produces new trajectories $\boldsymbol{T}(\tau)$ from the specified trajectory constraints and the shape descriptor model of the original trajectory. The reactive controller first determines a setpoint for the pose $\boldsymbol{T}_{d}$ and the pose derivative $\dot{\boldsymbol{T}}_{d}$ (velocity), based on an estimation of the progress $\tau_{c}$ along the trajectory and the current robot pose $\boldsymbol{T}_{c}$. The task specification and control framework eTaSL then combines trajectory following with other reactive behaviors to be executed on the robot using velocity-resolved control.

This adaptation preserves the shape of the original trajectory through the usage of coordinate-invariant shape descriptors, resulting in good generalization properties. The second contribution is the integration of the invariant trajectory generator into the task specification and control framework eTaSL. The resulting controller enables the combination of trajectory following with other reactive behaviors such as collision avoidance and force interaction with a human, possibly specified in joint or null space. Progress along the trajectory is estimated on-line for determining the appropriate setpoints and also fed back to the shape-preserving trajectory generator.

The remainder of this paper is structured as follows. Section II reviews related work, while Section III reviews the fundamentals of invariant shape descriptors. Section IV introduces the new approach for adapting end-effector trajectories in a shape-preserving way, while Section V explains the integration with the reactive controller for trajectory following. Section VI illustrates the invariant properties of the approach and shows how trajectories are adapted and followed towards a moving target including obstacle avoidance. Section VII provides a discussion of the results.

\section{RELATED WORK}

Different methods are available in Learning by Demonstration to generalize and adapt demonstrated motion trajectories towards new situations. Probabilistic approaches such as Gaussian Mixture Models [5], Probabilistic Movement Primitives [6] or Probabilistic Principal Component Analysis [7] try to capture the nominal task behavior together with its variability in probability distributions. This enables knowing what features are most important but comes with the downside that multiple demonstrations are required and that extrapolation outside of the set of demonstrations is not well defined.

Dynamical system approaches such as Dynamic Movement Primitives [8] or Stable Estimator of Dynamical Systems [9] adapt trajectories by calculating appropriate forcing terms in dynamical attractor systems. The models can be learned from one demonstration or from several demonstra- tions using various statistical techniques. They are fast and show great similarity during adaptation. However, the quality of the reproduction typically deteriorates when moving farther away in state space from the demonstrations.

To improve generalization farther away in state space, it is possible to apply global transformations on the trajectory, such as Euclidean [10] or affine [11] transformations to preserve invariant trajectory features. However, it is possible that no transformation exists that satisfies all new trajectory constraints at the same time, so that trajectory deformation is required. To calculate appropriate deformations, trajectory optimization is often used. Commonly, local deformations of the trajectory are sought that minimize the geometric distance between the adapted and original trajectory, for example, using splines [12]. The choice of distance metric can be further generalized to a problem over a Hilbert space [13]. Instead of the trajectory distance, the motion profile (or its derivatives) can also be preserved as the characteristic motion features [14]. This paper's method, based on [3], fits in the latter trajectory optimization category, but instead of using a norm in Cartesian space, we try to minimize a norm based on a coordinate-invariant description of the shape allowing for better generalization in different regions of the state space.

\section{PRELIMINARIES ON INVARIANT DESCRIPTORS}

To represent a rigid-body trajectory, a body reference frame $\{o b j\}$ is typically attached to the rigid body, of which the translation and rotation with respect to a world reference frame $\{w\}$ is described using a position vector ${ }_{w}^{o b j} \boldsymbol{p}$ and a rotation matrix ${ }_{w}^{o b j} \boldsymbol{R}$ (Figure 2a). However, the trajectory coordinates in ${ }_{w}^{o b j} \boldsymbol{p}$ and ${ }_{w}^{o b j} \boldsymbol{R}$ depend on the specific choice of reference frames $\{o b j\}$ and $\{w\}$. Learning motion models from these coordinates would limit the model to the chosen reference frames, restricting its ability to generalize.

Trajectory shape descriptors on the other hand represent trajectories in a coordinate-invariant way by describing local changes in the trajectory in terms of differential-geometric properties. These changes are defined and expressed in local moving frames that are unambiguously characterized by the motion itself. Multiple types of shape descriptors exist. This 


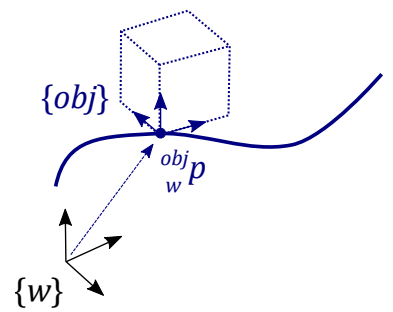

(a) Object trajectory

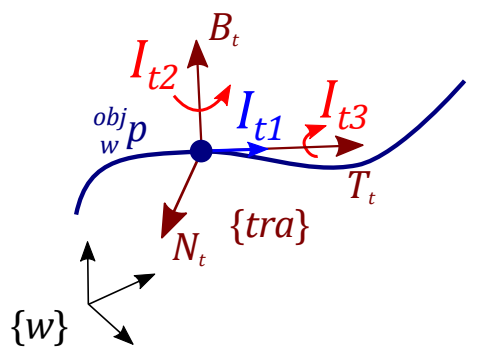

(b) Translation invariants $I_{t 1}, I_{t 2}$ and $I_{t 3}$

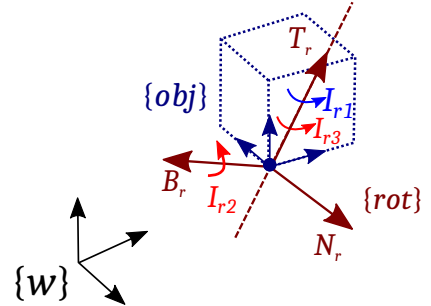

(c) Rotation invariants $I_{r 1}, I_{r 2}$ and $I_{r 3}$

Fig. 2: Definition of the extended Frenet-Serret invariant (eFSI) shape descriptor for representing rigid-body trajectories in a coordinate-free way. (a) Motion trajectory of a rigid body $\{o b j\}$ with respect to $\{w\}$. (b) The translation is represented by three invariants $I_{t 1}, I_{t 2}$ and $I_{t 3}$ in the local moving translational Frenet-Serret frame $\{t r a\}$. (c) The rotation is represented by three invariants $I_{r 1}, I_{r 2}$ and $I_{r 3}$ in the local moving rotational Frenet-Serret frame $\{$ rot $\}$.

paper relies on the extended Frenet-Serret invariants (eFSI) for rigid-body motion [3].

\section{A. Definition of the extended Frenet-Serret Invariants (eFSI)}

The object's translation and rotation are described separately for the extended Frenet-Serret invariants.

1) Object translation: Suppose the object's translation in the world $\{w\}$ is given by the translational velocity vector ${ }_{w}^{o b j} \dot{\boldsymbol{p}}(\tau)$, where $\tau$ is a progress parameter along the trajectory, for example, time or arc length. The translational Frenet-Serret invariants are then defined in a local moving frame, referred to as the translational Frenet-Serret frame $\{t r a\}$, visualized in Figure $2 \mathrm{~b}$. The first normalized axis $\boldsymbol{T}_{t}$ of this frame lies along the direction of ${ }_{w}^{o b j} \dot{\boldsymbol{p}}(\tau)$, i.e. the tangent to the point trajectory. The second normalized axis $\boldsymbol{B}_{t}$ lies along the rotational axis by which the tangent is instantaneously rotating (sometimes called the binormal). The third axis $N_{t}$ (the normal) follows from the definition of the first two axes, since it is an orthogonal frame.

Representing the frame's orientation using ${ }_{w}^{\operatorname{tra}} \boldsymbol{R}(\tau)$, the change in orientation ${ }_{w}^{\operatorname{tra}} \boldsymbol{R}^{\prime}(\tau)$ is then defined as follows using two invariant functions $I_{t 2}(\tau)$ and $I_{t 3}(\tau)$ :

$$
{ }_{w}^{t r a} \boldsymbol{R}^{\prime}(\tau)={ }_{w}^{t r a} \boldsymbol{R}(\tau)\left[\begin{array}{ccc}
0 & 0 & I_{t 2}(\tau) \\
0 & 0 & -I_{t 3}(\tau) \\
-I_{t 2}(\tau) & I_{t 3}(\tau) & 0
\end{array}\right]
$$

The change in translation of the object ${ }_{w}^{o b j} \boldsymbol{p}^{\prime}(\tau)$ is defined using another invariant $I_{t 1}$ :

$$
{ }_{w}^{o b j} \boldsymbol{p}^{\prime}(\tau)={ }_{w}^{t r a} \boldsymbol{R}(\tau)\left(\begin{array}{c}
I_{t 1}(\tau) \\
0 \\
0
\end{array}\right) .
$$

Equation (1) is more commonly known as the Frenet-Serret differential equations [15], though note we use a different ordering of the second and third axis to be consistent with other shape descriptor representations [3].

If the progress parameter $\tau$ corresponds to time, then $I_{t 1}$ becomes the magnitude of the translational velocity vector and $I_{t 2}$ and $I_{t 3}$ signify rotational velocities of the FrenetSerret frame $\{t r a\}$. If the progress parameter $\tau$ corresponds to the arc length $s$, then $I_{t 2}$ and $I_{t 3}$ are related to the curvature and torsion along the trajectory and $I_{t 1}$ is a constant equal to the total trajectory length $L$.

2) Object rotation: The rotational part of the trajectory is handled analogously to the translation, but now starting from the rotational velocity vector ${ }_{w}^{o b j} \boldsymbol{\omega}$. The rotational FrenetSerret invariants are defined in another local moving frame, referred to as the rotational Frenet-Serret frame $\{$ rot $\}$ (see Figure 2c). The first axis of this frame lies along the direction of ${ }_{w}^{o b j} \boldsymbol{\omega}$. The second and third axes are defined similarly as for $\{t r a\}$. Representing the orientation of $\{r o t\}$ using ${ }_{w}^{r o t} \boldsymbol{R}(\tau)$, the change in orientation ${ }_{w}^{r o t} \boldsymbol{R}^{\prime}(\tau)$ of this frame is defined as a function of two invariants $I_{r 2}(\tau)$ and $I_{r 3}(\tau)$ :

$$
{ }_{w}^{r o t} \boldsymbol{R}^{\prime}(\tau)={ }_{w}^{r o t} \boldsymbol{R}(\tau)\left[\begin{array}{ccc}
0 & 0 & I_{r 2}(\tau) \\
0 & 0 & -I_{r 3}(\tau) \\
-I_{r 2}(\tau) & I_{r 3}(\tau) & 0
\end{array}\right] .
$$

The change in object orientation ${ }_{w}^{o b j} \boldsymbol{R}^{\prime}(\tau)$ is finally defined using another invariant $I_{r 1}(\tau)$ :

$$
{ }_{w}^{o b j} \boldsymbol{R}^{\prime}(\tau)={ }_{w}^{r o t} \boldsymbol{R}(\tau)\left[\begin{array}{ccc}
0 & 0 & 0 \\
0 & 0 & -I_{r 1}(\tau) \\
0 & I_{r 1}(\tau) & 0
\end{array}\right]{ }^{o b j} \boldsymbol{R}(\tau) .
$$

This equation signifies a rotation $I_{r 1}(\tau)$ of the object around the tangent of $\{r o t\}$, which is related to the object frame using the final term ${ }_{\text {rot }}^{\text {obj }} \boldsymbol{R}$. This term holds the object orientation $\{a b j\}$ with respect to the rotational Frenet-Serret frame $\{$ rot $\}$.

To summarize, the rigid-body trajectory can be represented using a complete shape descriptor I consisting of six functions: $I_{t 1}, I_{t 2}, I_{t 3}, I_{r 1}, I_{r 2}$, and $I_{r 3}$. For calculating the shape descriptor, analytical formulas can be used. However, in practice, these formulas suffer from noise sensitivity and the influence of singularities where certain invariants become undefined. In [16], a numerical calculation approach was proposed to deal with these problems.

\section{B. Reconstruction of the trajectory from the shape descriptor}

To reconstruct the original trajectory, the generative equations (1) - (4) need to be integrated from the shape descriptor. Generally, no closed-form solution exists for the reconstructed trajectory. Therefore, we discretize the trajectory 
in $N$ samples with step size $\Delta \tau$. To simplify notations, we introduce for each sample $k$, a variable referred to as the trajectory state $\boldsymbol{X}_{k}$ that holds all information related to the object's position ${ }_{w}^{o b j} \boldsymbol{p}_{k}$ and orientation ${ }_{w}^{o b j} \boldsymbol{R}_{k}$, and the moving Frenet-Serret frames ${ }_{w}^{t r a} \boldsymbol{R}_{k}$ and ${ }_{w}^{r o t} \boldsymbol{R}_{k}$ at sample $k$ :

$$
\boldsymbol{X}_{k}=\left({ }_{w}^{t r a} \boldsymbol{R}_{k},{ }_{w}^{o b j} \boldsymbol{p}_{k},{ }_{w}^{\text {rot }} \boldsymbol{R}_{k},{ }_{w}^{o b j} \boldsymbol{R}_{k}\right) .
$$

Likewise, we introduce a variable for the invariant inputs $\mathbf{I}_{k}$ :

$$
\mathbf{I}_{k}=\left(I_{t 1}[k], I_{t 2}[k], I_{t 3}[k], I_{r 1}[k], I_{r 2}[k], I_{r 3}[k]\right) .
$$

Now, an integrator function $\boldsymbol{F}$ can be defined to integrate $\boldsymbol{X}_{k+1}$ from $\boldsymbol{X}_{k}$ using $\mathbf{I}_{k}$ over the integration period $\Delta \tau$ :

$$
\boldsymbol{X}_{k+1}=\boldsymbol{F}\left(\boldsymbol{X}_{k}, \mathbf{I}_{k}, \Delta \tau\right)
$$

This integration is exact when $\mathbf{I}_{k}$ is assumed piecewise constant and Rodrigues' rotation formula is used to correctly deal with the orientation dynamics. Explicit expressions of this rotation formula can be found in [17].

Provided we supply initial values $\boldsymbol{X}_{0}$ of the trajectory state, the whole trajectory can now be reconstructed using the procedure outlined in Algorithm 1.

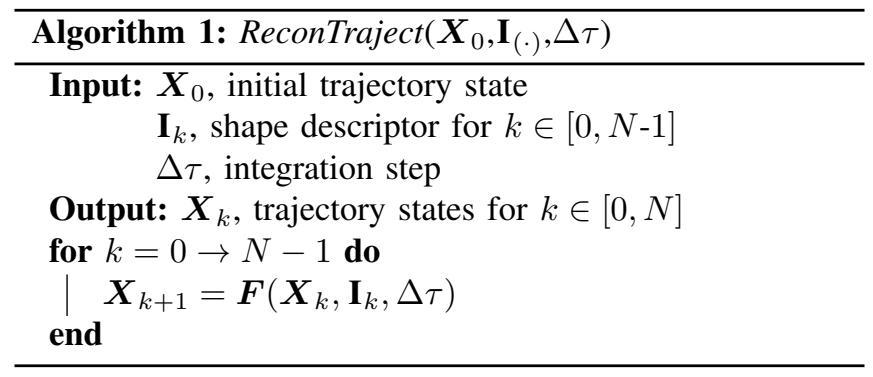

\section{SHAPE-PRESERVING TRAJECTORY ADAPTATION}

The trajectory reconstruction procedure outlined in Algorithm 1 is now used to formulate a method that allows online adaptation of the generated trajectory to fulfill new task requirements (e.g. adapting to a different target position and orientation). The objective is to preserve the overall shape of the trajectory as much as possible which is represented by the shape descriptor model $\mathbf{I}^{\text {model }}$.

The adaptation method relies on the generative equations summarized in (5). These may be interpreted as a dynamical system $\boldsymbol{F}$ with states $\boldsymbol{X}$ (holding trajectory information) and controls I (containing the values of the shape descriptor). By allowing changes in the invariant values $\mathbf{I}$, a different trajectory $\boldsymbol{X}$ can be obtained after reconstruction. To find the necessary changes that result in the desired trajectory, a constrained optimal control problem is formulated.

The optimization problem can be formulated over the whole horizon in which the trajectory takes place, but solving this may take an excessive amount of time if the number of trajectory samples $N$ is large. Therefore, we employ a receding window approach with window size $M<<N$. This is also inspired by the observation that changes in the shape descriptor have more influence on the reconstructed trajectory when they occur earlier, due to the double integration when reconstructing (1) - (2) and (3) - (4). Another

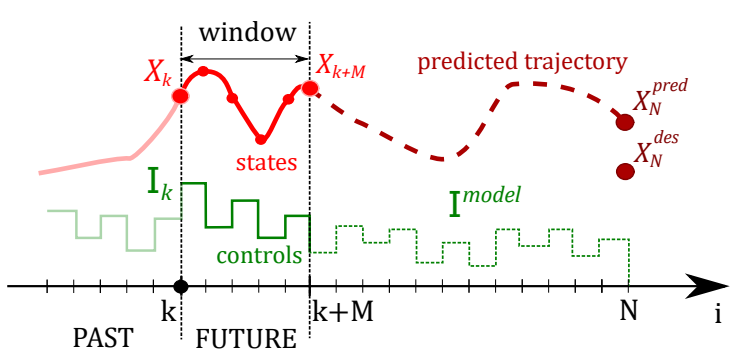

Fig. 3: Illustration of the receding window approach. Starting from the current state $\boldsymbol{X}_{k}$, a new trajectory needs to be found that goes to the desired target state $\boldsymbol{X}_{N}^{\text {des }}$. The trajectory is locally adapted through changes of the invariant control inputs $\mathbf{I}_{k}$ in the local window $[k k+M]$. The target state $\boldsymbol{X}_{N}^{\text {des }}$ is related to the window by reconstructing the trajectory using the shape descriptor of the original trajectory $\mathbf{I}^{\text {model }}$. The deviation between the predicted state $\boldsymbol{X}_{N}^{\text {pred }}$ and the desired state $\boldsymbol{X}_{N}^{\text {des }}$ is minimized in the optimization problem.

argument for the window approach is that it does not make sense to calculate shape adjustments in the later part of the trajectory since conditions may still change.

\section{A. Errors considered in the objective function}

The objective function of the optimization problem consists of costs that are related to the following errors.

1) Shape error: To ensure that the shape of the generated trajectory is preserved with respect to the original trajectory, the $6 \times 1$ error vector $\Delta \mathbf{I}_{k}$ between the shape descriptor $\mathbf{I}_{k}$ of the generated trajectory and the shape descriptor of the original trajectory $\mathbf{I}_{k}^{\text {model }}$ is considered for each sample $k$ :

$$
\Delta \mathbf{I}_{k}=\mathbf{I}_{k}-\mathbf{I}_{k}^{\text {model }} .
$$

2) Reach target state error: The main challenge with formulating the trajectory generation problem using a receding window is to relate the optimization variables in the window to the desired target state $\boldsymbol{X}_{N}^{\text {des }}$, which lies outside of the window in the future. The target state contains the desired position and orientation of the object at the end of the trajectory, but may also contain the desired orientations of the Frenet-Serret frames $\{t r a\}$ and $\{r o t\}$. The practical significance of the latter is that it allows specifying the direction of the tangent plus the tangent's change in direction at the end of the trajectory for both translation and rotation.

To relate the target state with the window, we consider the last trajectory state in the window, $\boldsymbol{X}_{k+M}$, and reconstruct the remainder of the trajectory with Algorithm 1 and using the shape descriptor model $\mathbf{I}^{\text {model }}$ of the original trajectory:

$$
\boldsymbol{X}_{k+M: N}^{\text {pred }}=\text { ReconTraject }\left(\boldsymbol{X}_{k+M}, \mathbf{I}_{k+M: N}^{\text {model }}, \Delta \tau\right) .
$$

Figure 3 provides an illustration of the procedure that relates the target state to the window through the invariant shape descriptor model. The result of this reconstruction is a prediction $\boldsymbol{X}^{\text {pred }}$ of the remainder of the trajectory after the window. The last sample $\boldsymbol{X}_{N}^{\text {pred }}$ of this prediction should correspond to the desired target state $\boldsymbol{X}_{N}^{\text {des }}$. The error 
between the two is notated as follows and added to the objective function:

$$
\Delta \boldsymbol{X}_{N}=\boldsymbol{X}_{N}^{p r e d}-\boldsymbol{X}_{N}^{\text {des }}
$$

where the errors on the three rotation matrices in $\Delta \boldsymbol{X}_{N}$ are each formulated as $\Delta \boldsymbol{R}_{N}=\left(\boldsymbol{R}_{N}^{\text {des }}\right)^{T} \boldsymbol{R}_{N}^{\text {pred }}-\boldsymbol{I}_{3}$.

\section{B. Receding window optimization for trajectory adaptation}

The complete optimization problem for generating shapepreserving trajectories starting from the shape descriptor of the model trajectory is formulated as follows:

$$
\begin{array}{lll}
\underset{\boldsymbol{X}_{(\cdot)}, \mathbf{I}_{(\cdot)}, L, \Theta}{\operatorname{minimize}} \sum_{i=k}^{k+M}\left(\left\|\Delta \mathbf{I}_{i}\right\|_{W_{1}}^{2}\right)+\left\|\Delta \boldsymbol{X}_{N}\right\|_{W_{2}}^{2} \\
\text { s.t. } & \boldsymbol{X}_{k}=\boldsymbol{X}_{k}^{\text {curr }} & \\
& \boldsymbol{X}_{i+1}=\boldsymbol{F}\left(\boldsymbol{X}_{i}, \mathbf{I}_{i}, \Delta \tau\right) & \forall i \in[k, k+M-1] \\
& I_{t 1}^{\text {model }}[i]=L & \forall i \in[k, N] \\
& I_{r 1}^{\text {model }}[i]=\Theta & \forall i \in[k, N]
\end{array}
$$

1) Objective function: The objective function consists of the weighted norm of the error terms (6) and (7). $W_{1}$ is a diagonal matrix that weights the importance of different invariants in the shape descriptor with respect to each other. The resulting cost $\left\|\Delta \mathbf{I}_{i}\right\|_{W_{1}}^{2}$ can be seen as the deformation energy necessary for bending and twisting the trajectory towards the new situation with $W_{1}$ being the stiffness parameters. $W_{2}$ represents four different weights related to the different parts in the target constraint $\boldsymbol{X}_{N}=$ $\left({ }_{w}^{o b j} \boldsymbol{p}_{N},{ }_{w}^{o b j} \boldsymbol{R}_{N},{ }_{w}^{t r a} \boldsymbol{R}_{N},{ }_{w}^{r o t} \boldsymbol{R}_{N}\right)$. Some of these weights may be put to zero if that particular part of the target constraint is unimportant.

2) Continuity constraint: Each time before solving the window, the first sample $k$ of the window and the corresponding trajectory state $\boldsymbol{X}_{k}^{\text {curr }}$ are determined from the previous solution using the current progress $\tau_{c} \in[0,1]$ along the trajectory, as estimated by the reactive controller (see Section V). By imposing the complete trajectory state $\boldsymbol{X}_{k}^{\text {curr }}$, there is second-order continuity in the generated trajectories. For the very first trajectory $(k=0)$ where the robot starts from standstill, it is possible to exclude the moving frames $\{t r a\}$ and $\{r o t\}$ from $\boldsymbol{X}_{0}^{\text {curr }}$, allowing the optimization the freedom to choose starting directions in translation and rotation that best align with the other constraints.

3) Scale changes: When $\tau$ signifies the geometric progress along the trajectory, the first two invariants $I_{r 1}$ and $I_{t 1}$ should be interpreted as two constant scales: the total length $L$ and the total rotated angle $\Theta$ along the trajectory. Constraints $(8 \mathrm{~d})-(8 \mathrm{e})$ are added for adapting the original scales in $I_{r 1}$ and $I_{t 1}$ of the model over the whole horizon, so that the predicted trajectory $\boldsymbol{X}^{\text {pred }}$ can be re-scaled outside of the window to reach the desired target state $\boldsymbol{X}_{N}^{\text {des }}$.

To summarize, the new trajectory is generated from the model trajectory shape descriptor by a local shape adaptation combined with a global change in scale.

\section{Solution of the optimization problem}

The optimal control problem (8a) - (8e) was implemented and solved using the CasADi framework [18]. The optimization problem is nonlinear and non-convex, so the solution may be a local one. In practice, the local solution suffices for well-chosen initial values. In the initial window, the states and controls are initialized with the trajectory and shape descriptor of the original trajectory. In subsequent windows, the solution of the previous step is used as initialization, typically resulting in only a few iterations and a significant speedup. The trajectory generator is stopped when deemed close enough to the target state $\boldsymbol{X}_{N}^{\text {des }}$ at which point the reactive controller (Section $\mathrm{V}$ ) will continue on its own.

\section{Adding the velocity profile}

The above optimization problem (8a) - (8e) produces a trajectory $\boldsymbol{X}(\tau)$ that is parameterized as a function of $\tau$, the progress along the trajectory. When $\tau$ is not time but signifies a geometric progress (e.g. arc length), the velocity profile $\dot{\tau}$ along the path is not yet specified. It can be chosen in multiple ways: for example, a uniform velocity profile, a minimum-jerk velocity profile or just re-applying the velocity profile of the original trajectory. Once chosen, the timederivative of the trajectory state $\dot{\boldsymbol{X}}(\tau)$ is found as follows: $\dot{\boldsymbol{X}}=\frac{d \boldsymbol{X}}{d \tau} \dot{\tau}$, where $\frac{d \boldsymbol{X}}{d \tau}$ is obtained from the results of the optimization problem. The time-derivative will be used by the reactive controller in Section $\mathrm{V}$ to find an appropriate velocity feed-forward.

\section{REACTIVE CONTROL USING ETASL}

The previous section explained how new trajectories are calculated. This section deals with the formulation of the robot controller that executes and follows this trajectory.

The trajectory following task is implemented using the constraint-based task specification and control framework eTaSL [4]. In eTaSL, each behavior $j$ is specified with a task function $e_{j}\left(\mathbf{q}, \chi_{f}, t\right)$, where $\mathbf{q}$ are the robot joint variables and $\chi_{f}$ are optional feature variables chosen to express task constraints in a more natural way. From this specification, eTaSL can automatically generate a controller in the following way. Each task function is imposed to evolve as a first-order dynamical system with time constant $\mathbf{K}^{-1}$ :

$$
\frac{d}{d t} e_{j}\left(\mathbf{q}, \chi_{f}, t\right)=-\mathbf{K} e_{j}\left(\mathbf{q}, \chi_{f}, t\right)
$$

Expanding the total time-derivative into partial derivatives captured in the Jacobian $\mathbf{J}_{j}$ yields the following control law:

$$
\mathbf{J}_{j}\left(\begin{array}{c}
\dot{\mathbf{q}} \\
\dot{\chi}_{f}
\end{array}\right)=-\mathbf{K} e_{j}-\frac{\delta e_{j}}{\delta t},
$$

where $-\mathbf{K} e_{j}$ should be interpreted as a feedback term, while $-\delta e_{j} / \delta t$ is a velocity feed-forward term that ensures good tracking behavior. Each desired behavior (e.g. trajectory following, force control, collision avoidance) has its own task function. The resulting control laws are then all combined as constraints in a quadratic optimization problem. Conflicts are resolved by adding slack variables $\epsilon_{j}$ in the control 
laws (10) which are weighted in the objective function of the optimization problem. Inequality constraints (e.g. joint position and velocity limits) are also possible.

The trajectory generator of Section IV provides a global trajectory $\boldsymbol{X}(\tau)$ that the robot controller should follow. From the complete trajectory state, we only consider the position and orientation of the object and represent these using the pose matrix $\boldsymbol{T}=\left[\begin{array}{cc}{ }^{o b j} \\ w & { }^{o b j} \boldsymbol{p} \\ \boldsymbol{O}_{1 \times 3} & 1\end{array}\right]$. The trajectory following constraint is then specified as: $\mathbf{e}=\boldsymbol{T}_{c}(\mathbf{q})^{-1} \boldsymbol{T}_{d}$, where $\boldsymbol{T}_{c}(\mathbf{q})$ is the current pose of the robot end effector and $\boldsymbol{T}_{d}$ is the desired setpoint for the pose. This setpoint is repeatedly determined by finding the trajectory point that lies closest to the end effector's current position. Only the position is used for determining the setpoint, the progress in rotation is therefore coupled to the progress in position. The corresponding progress $\tau_{c}$ along the trajectory is fed back to the trajectory generator. Velocity feed-forward in terms of the derivative of the trajectory $\dot{\boldsymbol{T}}_{d}$ is provided by the trajectory generator and used by eTaSL to improve dynamic tracking.

\section{RESULTS}

This section illustrates the generalization capabilities of the proposed trajectory adaptation approach. Furthermore, we show the reactive behavior of the controller for tracking the adapting trajectory in presence of obstacles.

\section{A. Trajectory adaptation to changing target pose}

We evaluate the trajectory adaptation towards new targets for our proposed approach, referred to as receding window optimization (Section IV), compared to the full horizon optimization in [3]. The trajectory is a scooping motion that was originally demonstrated by a human and recorded using the HTC Vive system. Figures $4 \mathrm{a}$ and $4 \mathrm{~b}$ depict the original trajectory in blue. Both the position and orientation of the target state are changed to 20 new locations in state space that are evenly distributed around the start state in the XYplane. In addition to the global change in the target state, there are small random changes in the target position during the motion, therefore requiring on-line adaptation.

For the first trajectory state $\boldsymbol{X}_{0}^{\text {curr }}$, set in (8b), the starting position ${ }_{w}^{o b j} \boldsymbol{p}_{0}$ and orientation ${ }_{w}^{o b j} \boldsymbol{R}_{0}$ were taken the same as the demonstration, while ${ }_{w}^{t r a} \boldsymbol{R}_{0}$ and ${ }_{w}^{r o t} \boldsymbol{R}_{0}$ were kept unconstrained, allowing the optimization to choose the direction in which it departs. Starting from the second trajectory, the first state is wholly enforced to remain equal to the previous solution, resulting in second-order continuity. Table I summarizes the choice of weight parameters for the trajectory generator in this experiment. For the receding window approach, the size of the window $M$ is chosen as 20 samples. For the full horizon approach, the effective horizon is between the current sample and the end of the trajectory.

Figure 4 visualizes 3 out of 20 adapted trajectories for the receding window and the full horizon method. Though the target positions are very different from the demonstration, the overall shape remains well-preserved for both methods,
TABLE I: Choice of weight parameters $W_{1}$ and $W_{2}$ in the optimization problem (8a) for trajectory adaptation.

\begin{tabular}{l|llllll}
$W_{1}$ & $I_{t 1}$ & $I_{t 2}$ & $I_{t 3}$ & $I_{r 1}$ & $I_{r 2}$ & $I_{r 3}$ \\
\hline & $10^{-2}$ & $10^{-4}$ & $10^{-4}$ & $10^{-3}$ & $10^{-4}$ & $10^{-4}$ \\
$W_{2}$ & ${ }^{o b j} \boldsymbol{p}_{N}$ & ${ }^{o b j} \boldsymbol{R}_{N}$ & ${ }_{w}^{t r a} \boldsymbol{R}_{N}$ & ${ }_{w}^{r o t} \boldsymbol{R}_{N}$ & & \\
\hline & 30.0 & 1.0 & 1.0 & 1.0 & &
\end{tabular}

which is also indicated by the corresponding shape descriptors in Figure 4c. Table II compares the average deviation of the shape descriptor from the demonstration over all trials, as well as the required calculation times. From the results, we can conclude that the receding window method has a slightly worse shape deviation compared to the full horizon method, but is still very comparable. The calculation time is a factor 7 faster for the receding window method, clearly showing its usefulness over the full horizon method.

\section{B. Trajectory adaptation and robot control for moving target}

The trajectory generator is now combined with the reactive eTaSL controller. Again, a task (S-shape) was demonstrated by a human and the parameters of the trajectory generator were chosen the same as before. The velocity profile $\dot{\tau}$ (see Section IV-D) is chosen to be preserved from the demonstration. During execution, the target position ${ }_{w}^{o b j} \boldsymbol{p}_{N}$ is linearly moved in Cartesian space, while the target orientation is also rotated linearly towards a new orientation ${ }_{w}^{o b j} \boldsymbol{R}_{N}$. (The final displacement in target position and rotation can be found in Figure 6 a at $\tau=1$.)

The reactive controller for trajectory following is demonstrated for the Universal Robots' UR10 manipulator. The kinematic model of the robot is automatically imported in eTaSL and includes joint position and velocity limit constraints, ensuring feasible control action on the real robot. The time constant $\mathbf{K}^{-1}$ for the trajectory following is set to $0.1 \mathrm{~s}$, and the controller operates at a frequency of $200 \mathrm{~Hz}$ sending desired joint velocities to the robot.

Figure 5 shows the robot near the start of the initial trajectory. During execution, the trajectory generator is continuously updating the reference trajectory towards the changing target, as can be seen in Figure 5b. The robot's trajectory is continuously adapted by the reactive controller towards the current reference trajectory until the end in Figure 5c. Figure 6 displays the trajectory coordinates and shape descriptors of the generated trajectories with respect to the demonstration. Deviations of the shape descriptor are kept small with respect to the original demonstration resulting in shape preservation. Remark that while the coordinateinvariant shape descriptor may experience discontinuities in the signals, the trajectory coordinates maintain second-order continuity thanks to the continuity constraint in (8c). Since the target is continuously moving away, the scales in $I_{r 1}$ and $I_{t 1}$ are increasing.

\section{Moving target including obstacle avoidance}

The previous case is extended with obstacle avoidance as an additional reactive behavior in the eTaSL controller. Static obstacles are better taken into account during the trajectory 
TABLE II: Comparison for the experiment in Section VI-A of the calculation time and the shape deviation between the shape-preserving trajectory generator optimizing over the full horizon [3] versus the receding window approach (8a) - (8e). The shape deviations are averaged over the final executed trajectory of all the 20 trials. The trajectory calculation time is averaged over all the intermediate trajectories for all trials. While the errors on the shape descriptors are comparable for both methods, the receding window approach is much faster by a factor of 7 .

\begin{tabular}{ll||ccc|ccc|c} 
& \multicolumn{1}{l||}{} & \multicolumn{2}{c|}{ deviation shape descriptor (translation) } & \multicolumn{3}{c|}{ deviation shape descriptor (rotation) } & calculation time \\
optimization & & $\Delta I_{t 1}[\mathrm{~m}]$ & $\Delta I_{t 2}\left[\frac{\mathrm{rad}}{-}\right]$ & $\Delta I_{t 3}\left[\frac{\mathrm{rad}}{-}\right]$ & $\Delta I_{r 1}[\mathrm{rad}]$ & $\Delta I_{r 2}\left[\frac{\mathrm{rad}}{-}\right]$ & $\Delta I_{r 3}\left[\frac{\mathrm{rad}}{-}\right]$ & per trajectory $[\mathrm{s}]$ \\
\hline full horizon & average & 0.0789 & 0.9995 & 0.9050 & 0.1495 & 2.4406 & 1.2135 & $\mathbf{0 . 2 3 1 0}$ \\
& std. dev. & $(0.0394)$ & $(0.3295)$ & $(0.4065)$ & $(0.1788)$ & $(1.9215)$ & $(1.2079)$ & $(0.1002)$ \\
\hline receding window & average & 0.0789 & 1.1136 & 0.6496 & 0.5414 & 2.5125 & 1.5905 & $\mathbf{0 . 0 3 1 2}$ \\
& std. dev. & $(0.0392)$ & $(0.3743)$ & $(0.2275)$ & $(0.4788)$ & $(1.1231)$ & $(0.9492)$ & $(0.0038)$
\end{tabular}

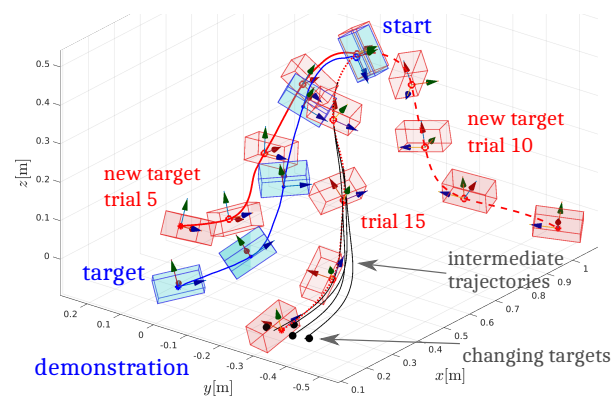

(a) Receding window trajectories $v$ s demonstration

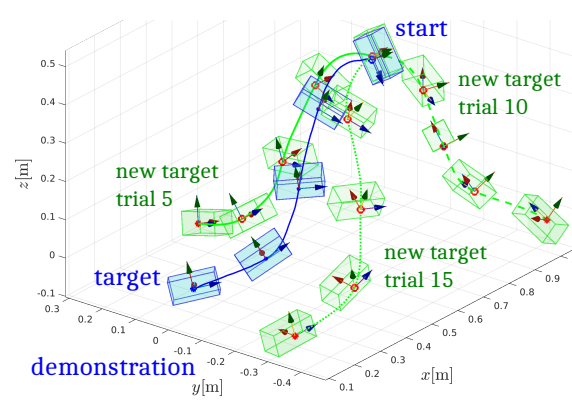

(b) Full horizon trajectories vs demonstration

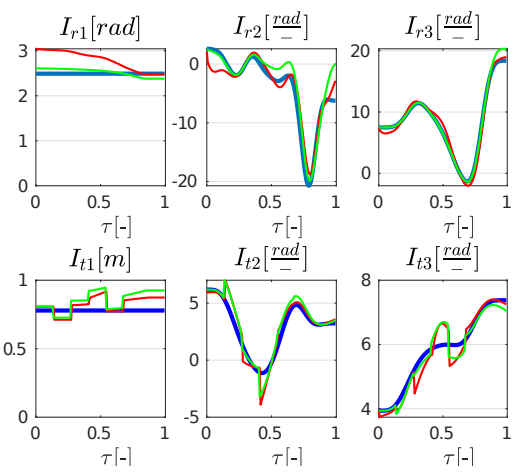

(c) Shape descriptors of trial 15 for both methods compared to model invariants (blue)

Fig. 4: Comparison between (a) the receding window approach and (b) the full horizon approach for shape-preserving trajectory adaptation of a demonstrated scooping motion. Only 3 out of 20 trials are depicted for each method in (a) and (b). During execution the desired target pose changes randomly in a small region. (c) In both approaches, the trajectories are adapted through corresponding (small) changes in the shape descriptor.

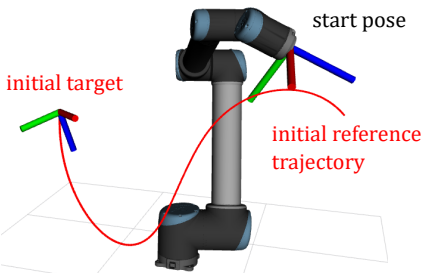

(a) Starting pose and initial trajectory

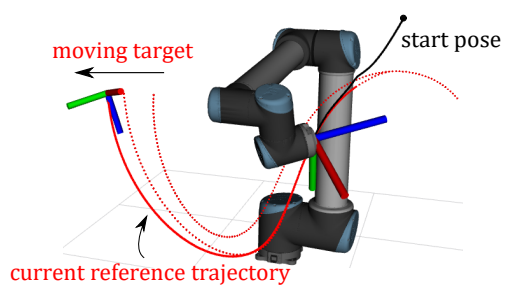

(b) Adaptation during execution

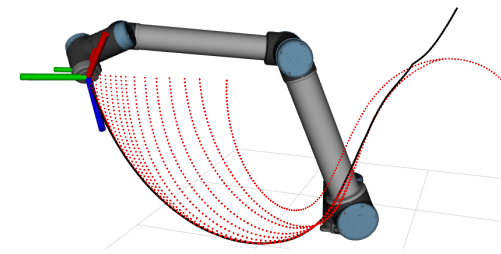

(c) Final executed trajectory

Fig. 5: Trajectory adaptation and control for a moving target (Section VI-B): the trajectory is being adapted towards a moving target that has a changing position and orientation. The dotted lines represent earlier calculated trajectories. The eTaSL reactive controller ensures a smooth transition between trajectories.

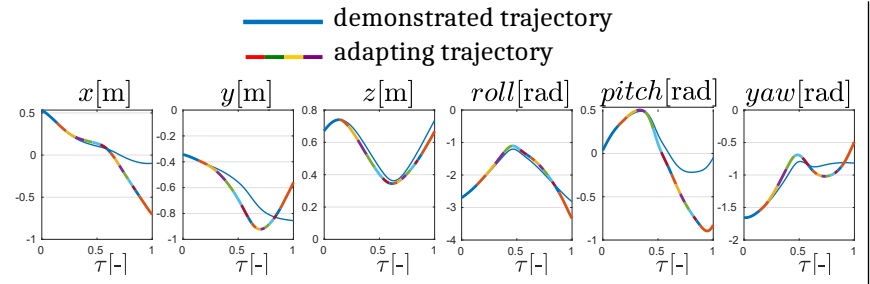

(a) Trajectory coordinates

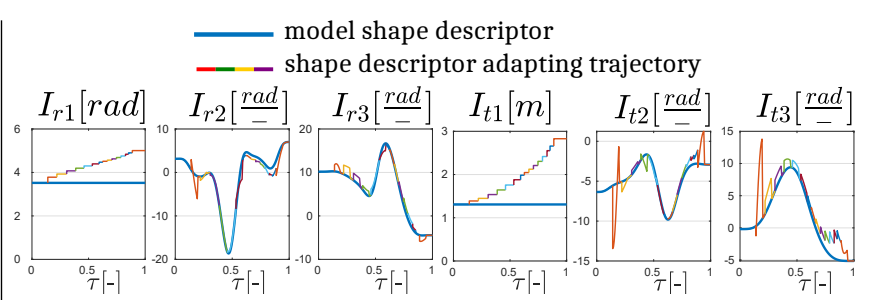

(b) Shape descriptors

Fig. 6: Trajectory adaptation and control for a moving target (Section VI-B): the trajectory coordinates and shape descriptors of the generated trajectories versus the initial demonstrated trajectory (in blue). A change in color signifies an adaptation of the calculated trajectory. The trajectories have second-order continuity even though the descriptors can be discontinuous. 


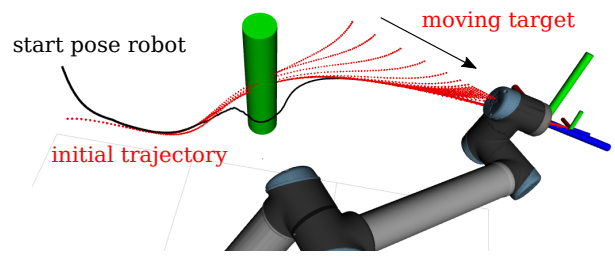

Fig. 7: Trajectory adaptation and following towards a moving target position and orientation. Additionally, the cylindrical obstacle needs to be avoided by the robot end effector.

generation since it will preserve the shape better. However, here we consider the obstacle as unknown during trajectory generation so that the reactive controller has to handle it.

Obstacle avoidance is implemented in eTaSL by adding a new task function $e=d$ (robot, obstacle $) \geq 0$, where robot and obstacle are modeled as two convex objects between which the distance $d$ should always remain positive to avoid collision. The corresponding control constant $\mathbf{K}$ determines the dynamics of the objects' interaction and is set here to $\mathbf{K}=2 \mathrm{~s}^{-1}$. The obstacle is modeled as a cylinder with radius $5 \mathrm{~cm}$ and set along the trajectory. The robot's end effector is modeled as a cube with width and length $6 \mathrm{~cm}$ and height $10 \mathrm{~cm}$. Figure 7 shows how the resulting trajectory is adapted correspondingly around the mantle of the cylinder.

\section{DisCUSSION AND CONCLUSIONS}

We have presented an approach for the on-line adaptation of end-effector trajectories where the overall shape of the original trajectory is preserved as much as possible. The trajectory generator is integrated with the eTaSL framework to achieve reactive sensor-based behaviors on the robot.

Compared to other trajectory deformation methods [10], [14], [13], the shape-preserving trajectory generator has no issues generalizing in different regions of the task space since a coordinate-free norm related to the shape deformation is minimized instead of a distance norm in Cartesian space. Another advantage is the flexibility with which trajectory constraints can be specified or not. For example, in Figure 4a the starting direction was not constrained, allowing the optimization to choose one that best preserves the shape.

The generated trajectory depends on the chosen parameters and weights in the optimization method. For example, a larger window size will result in a more smoothed out shape deformation. This raises the question of what parameters should be chosen to get the best solution. Generally, this depends on the context of the application where shape preservation is required. Therefore, the choice of weights should be seen as an opportunity to explore the solution space for a result that is satisfactory. The advantage of the approach is that it guarantees that the energy associated with the shape deformation is minimized given the chosen weights.

The advantage of using eTaSL as opposed to a standard impedance controller is the modular approach with which different sensor-based reactive behaviors can be combined, as demonstrated in Section VI-C. Furthermore, eTaSL is capable of tracking the progress along the trajectory itself. This work exploited that capability to integrate a global trajectory generator. In another work [7] using eTaSL, the progress along the trajectory was used to implement impedance behaviors along the trajectory, allowing for example to hold back and catch up the progress along the trajectory during physical human-robot interaction. While the reactive controller is not shape-preserving itself, this is not a real drawback since it typically performs only small corrections.

A possible extension of this work is to use an estimator to predict the target's motion. This would enable the trajectory generator to further improve the tracking behavior.

\section{REFERENCES}

[1] B. D. Argall, S. Chernova, M. Veloso, and B. Browning, "A survey of robot learning from demonstration," Robotics and autonomous systems, vol. 57, no. 5, pp. 469-483, 2009.

[2] A. Billard, S. Calinon, R. Dillmann, and S. Schaal, "Robot programming by demonstration," in Springer handbook of robotics. Springer, 2008, pp. 1371-1394.

[3] M. Vochten, T. De Laet, and J. De Schutter, "Generalizing demonstrated motion trajectories using coordinate-free shape descriptors," Robotics and Autonomous Systems, vol. 122, p. 103291, 2019.

[4] E. Aertbeliën and J. De Schutter, "etasl/etc: A constraint-based task specification language and robot controller using expression graphs," in 2014 IEEE/RSJ International Conference on Intelligent Robots and Systems, Sep. 2014, pp. 1540-1546.

[5] S. Calinon, F. Guenter, and A. Billard, "On learning, representing, and generalizing a task in a humanoid robot," Systems, Man, and Cybernetics, Part B: Cybernetics, IEEE Transactions on, vol. 37, no. 2, pp. 286-298, 2007.

[6] A. Paraschos, C. Daniel, J. Peters, and G. Neumann, "Probabilistic movement primitives," in Advances in Neural Information Processing Systems, 2013, pp. 2616-2624.

[7] C. A. V. Perico, J. De Schutter, and E. Aertbeliën, "Combining imitation learning with constraint-based task specification and control," IEEE Robotics and Automation Letters, vol. 4, no. 2, pp. 1892-1899, 2019.

[8] A. J. Ijspeert, J. Nakanishi, H. Hoffmann, P. Pastor, and S. Schaal, "Dynamical movement primitives: learning attractor models for motor behaviors," Neural computation, vol. 25, no. 2, pp. 328-373, 2013.

[9] S. M. Khansari-Zadeh and A. Billard, "Learning stable nonlinear dynamical systems with gaussian mixture models," Robotics, IEEE Transactions on, vol. 27, no. 5, pp. 943-957, 2011.

[10] K. Gräve and S. Behnke, "Incremental action recognition and generalizing motion generation based on goal-directed features," in Intelligent Robots and Systems (IROS), 2012 IEEE/RSJ International Conference on. IEEE, 2012, pp. 751-757.

[11] Q. C. Pham and Y. Nakamura, "A new trajectory deformation algorithm based on affine transformations," IEEE Transactions on Robotics, vol. 31, no. 4, pp. 1054-1063, Aug 2015.

[12] J. Aleotti and S. Caselli, "Robust trajectory learning and approximation for robot programming by demonstration," Robotics and Autonomous Systems, vol. 54, no. 5, pp. 409-413, 2006.

[13] A. D. Dragan, K. Muelling, J. A. Bagnell, and S. S. Srinivasa, "Movement primitives via optimization," in 2015 IEEE International Conference on Robotics and Automation (ICRA). IEEE, 2015, pp. 2339-2346.

[14] A. Pekarovskiy, T. Nierhoff, S. Hirche, and M. Buss, "Dynamically consistent online adaptation of fast motions for robotic manipulators," IEEE Transactions on Robotics, vol. 34, no. 1, pp. 166-182, Feb 2018.

[15] W. Kühnel, Differential geometry: curves-surfaces-manifolds. American Mathematical Soc., 2006, vol. 16.

[16] M. Vochten, T. De Laet, and J. De Schutter, "Robust optimizationbased calculation of invariant trajectory representations for point and rigid-body motion," in 2018 IEEE/RSJ International Conference on Intelligent Robots and Systems (IROS), 2018, pp. 5598-5605.

[17] R. M. Murray, S. S. Sastry, and L. Zexiang, A Mathematical Introduction to Robotic Manipulation, 1st ed. Boca Raton, FL, USA: CRC Press, Inc., 1994.

[18] J. A. E. Andersson, J. Gillis, G. Horn, J. B. Rawlings, and M. Diehl, "CasADi - A software framework for nonlinear optimization and optimal control," Mathematical Programming Computation, vol. 11, no. 1, pp. 1-36, 2019. 